\title{
The ketogenic diet for paediatric patients with super-refractory status epilepticus in febrile infection-related epilepsy syndrome
}

\author{
Xinhua Wang ${ }^{1}$, Xuan Gao ${ }^{2}$, Guoping Lu², Zhongying Lư ${ }^{3}$, Shuizhen Zhou'1 Yi Wang ${ }^{1}$ and Yuanfeng Zhou ${ }^{1 *}$
}

\begin{abstract}
Objective: To investigate the effect and safety of ketogenic diet (KD) for the treatment of paediatric patients with super-refractory status epilepticus (SRSE) in febrile infection-related epilepsy syndrome (FIRES).

Method: From January 1, 2015 to October 31, 2017, ten critically ill paediatric patients with SRSE in FIRES were included in this study and treated with KD. The treatment effects of KD were evaluated by using continuous encephalography (CEEG) and amplitude-integrated electro-encephalography (aEEG).

Results: All 10 patients fulfilled the diagnostic criteria of SRSE in FIRES and achieved ketosis within 24-72 $\mathrm{h}$ following the administration of KD. CEEG and aEEG were monitored for several weeks to assess the efficacy of KD on status epilepticus (SE). SE was contained in 8 patients within 2 to 19 days after initiation of KD, and KD was discontinued in the other 2 patients. One of the 10 patients demonstrated severe adverse effects.

Conclusion: KD may be an alternative and safe treatment option in critical paediatric patients with SRSE in FIRES.

Keywords: Ketogenic diet, Paediatric, Super-refractory status epilepticus, Febrile infection-related epilepsy syndrome
\end{abstract}

\section{Background}

Status epilepticus (SE) is one of the most life-threatening medical emergencies in children. Super-refractory status epilepticus (SRSE) is defined as an epileptic status that continues or recurs $24 \mathrm{~h}$ or more after the initiation of anaesthetic therapy, including reduction or withdrawal of anaesthetic agents [1]. The mortality of SRSE ranges from $23-85 \%$ [2-4], and current therapies demonstrate various drawbacks in treatment outcomes, adverse effects, and intolerance. Therefore, there is an urgent need to develop alternative or novel therapies to facilitate clinical management.

Ketogenic diet (KD) is a high fat, adequate protein, low carbohydrate diet, and it is currently recommended as a

\footnotetext{
*Correspondence: yuanfengzhou99@163.com

'Department of Neurology, Children's Hospital of Fudan University, Shanghai 201102, China

Full list of author information is available at the end of the article
}

safe and effective dietary treatment for chronic refractory epilepsy (infantile spasm, tuberous sclerosis, Doose syndrome, Dravet syndrome and focal epilepsy) in children. Recent reports indicate that KD is effective for refractory SE. We examined the efficacy and safety of KD in 10 children with SRSE in febrile infection-related epilepsy syndrome (FIRES) and used continuous encephalography (CEEG) and amplitude-integrated electro-encephalography (aEEG) to monitor and evaluate treatment and outcome.

\section{Patients and methods \\ Patients}

A total of 10 children (4 boys and 6 girls, age range 513 years, median 9 years) fulfilled the diagnostic criteria of FIRES at Children's Hospital of Fudan University from January 1, 2015 to October 31, 2017. FIRES is a sub-type of new-onset refractory status epilepticus (NORES) that occurs after a prior febrile infection, with

(c) The Author(s). 2020 Open Access This article is licensed under a Creative Commons Attribution 4.0 International License, which permits use, sharing, adaptation, distribution and reproduction in any medium or format, as long as you give appropriate credit to the original author(s) and the source, provide a link to the Creative Commons licence, and indicate if changes were made. The images or other third party material in this article are included in the article's Creative Commons licence, unless indicated otherwise in a credit line to the material. If material is not included in the article's Creative Commons licence and your intended use is not permitted by statutory regulation or exceeds the permitted use, you will need to obtain permission directly from the copyright holder. To view a copy of this licence, visit http://creativecommons.org/licenses/by/4.0/. 
onset of fever from $24 \mathrm{~h}$ to 2 weeks earlier than the onset of refractory SE. There may be no fever at the onset of SE. Patients in different age have similar course of disease [5]. The 10 patients began KD and were followed up for at least one year. Nine patients had no significant personal or family seizure histories, and one patient had 3 febrile seizures at age 5 . All patients had normal health and psychomotor development originally. All patients were assessed by Glasgow Coma Scale (GCS). All patients underwent cerebrospinal fluid (CSF) tests, including routine test, biochemical and culture tests, haemoculture test, and other body fluid culture test for infective pathogens. All patients underwent magnetic resonance imaging (MRI) examinations at $1.5 \mathrm{~T}$ (including T1WI, T2WI, and DWI). Nine out of ten patients tested autoimmune antibodies in CSF and blood. Haematuria tandem mass spectrometry was used to exclude the cases with absolute contraindications of KD. A 2742-gene panel targeted sequencing was tested in six out of ten patients. Clinical features of all patients are described in Table 1.

\section{KD scheme}

Prior to KD, all patients were screened for contraindications to KD therapy and fasted for 3 days to facilitate a faster transition to ketosis. The KD' grams rate (fat to combined carbohydrate and protein) was 4:1 for initiating the diet. The KD was administered via a gastric tube, and glucose-free medications were given. Finger-prick blood glucose and beta-hydroxybutyrate (BHB) levels were tested every $12 \mathrm{~h}$. Serum electrolytes, body weight, and details of intake-output were recorded. After the KD was initiated, the regimens of original anti-epileptic and anaesthetic drugs was not changed until the KD was proven effective.
The improvement in seizure frequency was divided into three categories: effective (seizure-free), partial effective (50-90\% improvement in seizure frequency) and ineffective ( $<50 \%$ improvement in seizure frequency). SE was also evaluated according to this criterion.

\section{EEG}

EEG electrodes were applied to the scalps according to the international 10-20 systems of electrode placement. The Nicolet Video-EEG (VEEG) monitor (Nicoletone, Middleton, Wisconsin, United States) was used for conventional EEG recordings. This device had a real-time and synchronous aEEG display software.

aEEG was derived from a double-channel recorder (F3-F4, C3-C4) raw EEG and displayed on a conventional semi-logarithmic scale. The signal was compressed to a rate of $6 \mathrm{~cm} / \mathrm{h}$.

Diagnostic criteria of seizures and SE via aEEG: Epileptic seizure activity reflected in the aEEG is generally regarded as a sudden rise of the minimum amplitude and a simultaneous rise of the maximum amplitude, usually followed by a short-term decreased amplitude. SE activity in the aEEG is generally seen as a saw-tooth pattern or an increase in the minimum and maximum amplitude that continued for over $30 \mathrm{~min}$ [6].

\section{EEG scheme}

After SE was confirmed by using conventional multichannel CEEG and aEEG monitoring for $72 \mathrm{~h}$, a dualchannel recording of CEEG and aEEG monitoring was used for a real-time evaluation of the KD' effects until SE began to be controlled and a normal background band appeared on the aEEG. Then, conventional multichannel CEEG monitoring was used for another $72 \mathrm{~h}$ to clarify whether the KD controlled convulsive status

Table 1 The demographic data of the patients

\begin{tabular}{|c|c|c|c|c|c|c|c|c|}
\hline No. & Age $(y)$ & Sex & Epilepsy history & Brain MRI & $\begin{array}{l}\text { Autoimmune antibodies } \\
\text { of blood and CSF }\end{array}$ & Gene test & GCS & SE type \\
\hline Case 1 & 10 & M & No & Bi-thalamus T2 hyperintensity & Not Available & Not Available & 8 & CSE/NCSE \\
\hline Case 2 & 13 & $\mathrm{~F}$ & No & Normal & Negative & Not Available & 11 & NCSE \\
\hline Case 3 & 9 & M & 3 times FS in 5 years old & Bi-hippocampus T2 hyperintensity & Negative & Negative & 10 & CSE/NCSE \\
\hline Case 4 & 8 & $\mathrm{~F}$ & No & $\begin{array}{l}\text { Bi-hippocampus, thalamus, frontal } \\
\text { and temporal lobe } \mathrm{T} 2 \text { hyperintensity }\end{array}$ & Negative & Negative & 7 & CSE/NCSE \\
\hline Case 5 & 5 & $\mathrm{~F}$ & No & Bi-hippocampus T2 hyperintensity & Negative & Not Available & 11 & CSE/NCSE \\
\hline Case 6 & 10 & M & No & Normal & Negative & Not Available & 10 & CSE/NCSE \\
\hline Case 7 & 9 & M & No & Normal & Negative & Negative & 8 & NCSE \\
\hline Case 8 & 7 & $\mathrm{~F}$ & No & $\begin{array}{l}\text { Bi-thalamus, frontal and parietal lobe } \\
\text { T2 hyperintensity }\end{array}$ & Negative & Negative & 10 & CSE/NCSE \\
\hline Case 9 & 5 & $\mathrm{~F}$ & No & Normal & Negative & Negative & 10 & CSE/NCSE \\
\hline Case 10 & 11 & $\mathrm{~F}$ & No & Bi-frontal T2 hyperintensity & Negative & Negative & 8 & CSE/NCSE \\
\hline
\end{tabular}

FS febrile seizure, GCS Glasgow Coma Scale, ICU intensive care unit, MRI magnetic resonance imaging, CSF cerebrospinal fluid, CSE convulsive status epilepticus, NCSE nonconvulsive status epilepticus 
epilepticus (CSE) or nonconvulsive status epilepticus (NCSE; criteria for the diagnosis of NCSE [7]). According to our EEG scheme, $72 \mathrm{~h}$ CEEG and aEEG monitoring were carried several times during 2-3 weeks.

\section{Results}

\section{EEG changes}

EEG recordings performed before the KD showed multifocal interictal epileptic discharge, which was consistent with SE that originated from focal areas and migrated over large areas of unilateral or bilateral hemispheres. SE in the aEEG was depicted as the repetitive or one continuous increase of the lower and upper margins.

For example, in case 2, conventional EEG confirmed continuous electrographic seizures arising from a focal region with a second generalization and no obvious clinical manifestations, which satisfied the diagnostic criteria of NCSE. The typical saw-tooth pattern was shown on aEEG trend (Fig. 1).

\section{MRI changes}

Early MRI showed abnormalities in 6 of the 10 patients (Table 1). The brain areas involved included thalami, hippocampi, frontal lobes, temporal lobes, and parietal lobes (Fig. 2). Brain atrophies ranged from mild to moderate severe levels were observed in nine patients at the 6-month follow-up (Table 2).

\section{Effects of the KD}

SE was controlled effectively in clinical and on EEG evaluations in eight patients after 2-19 days administration of KD with no recurrence of SE for more than 6 months, and the time to maintain seizure free was 15 days to 5 months. In 7 of them, KD safely kept for 3-12 months. In case $3, \mathrm{SE}$ was effectively controlled after 15 days of treatment with $\mathrm{KD}$, but KD was discontinued because of arrhythmia that occurred on the 27th days. However, seizure free still went on for 4 months even if KD was stopped. In addition, KD was ineffective in the other two patients and was discontinued. It should be noted that seizures reappeared in 7 patients after $\mathrm{KD}$ in a minimum of 15 days and a maximum of 5 months and developed into refractory epilepsy.

Regarding the side effects of $\mathrm{KD}$, urinary stones occurred in 3 cases and hematuresis in 1 case were observed in our study. However, the symptoms were quite

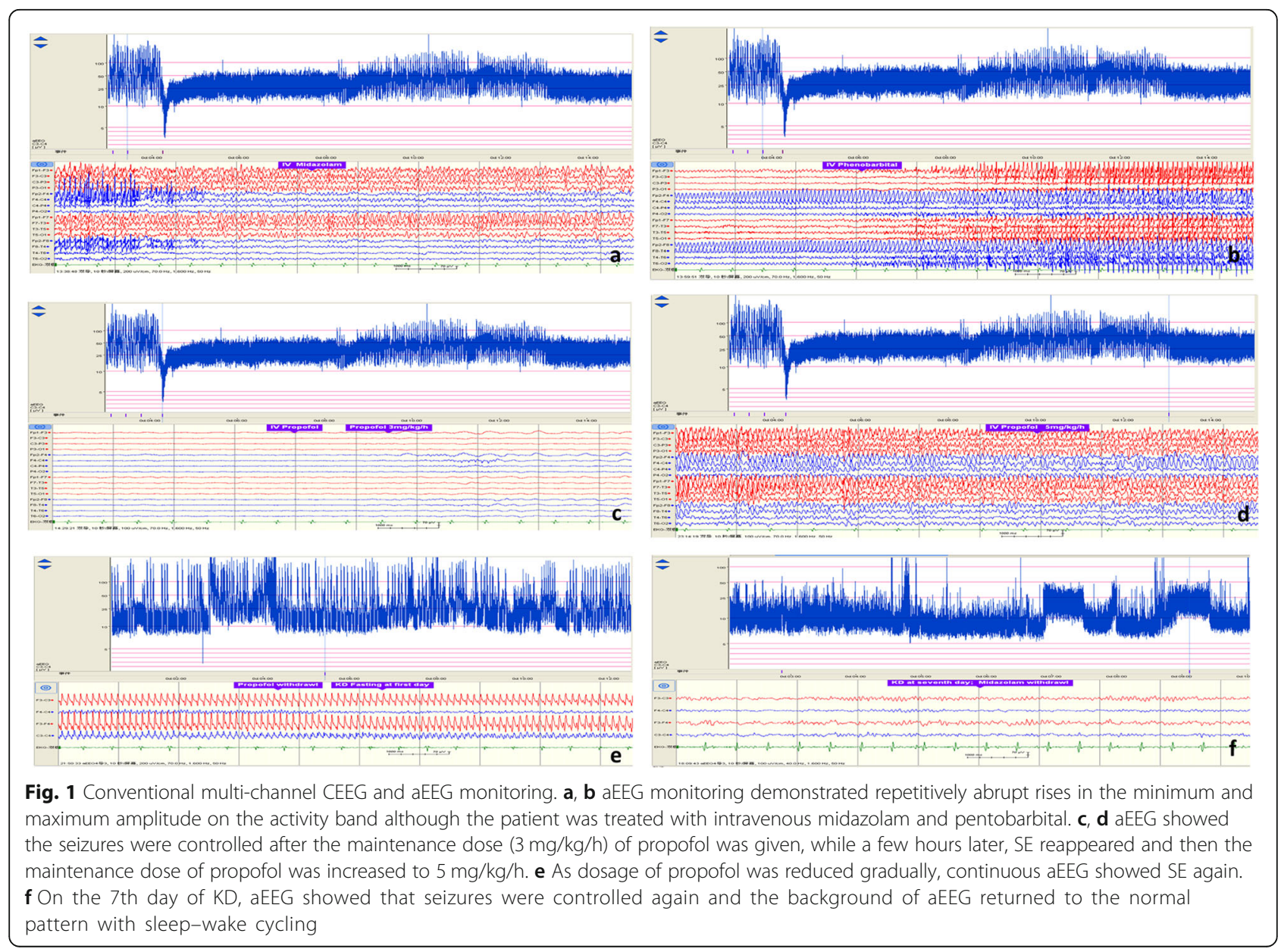




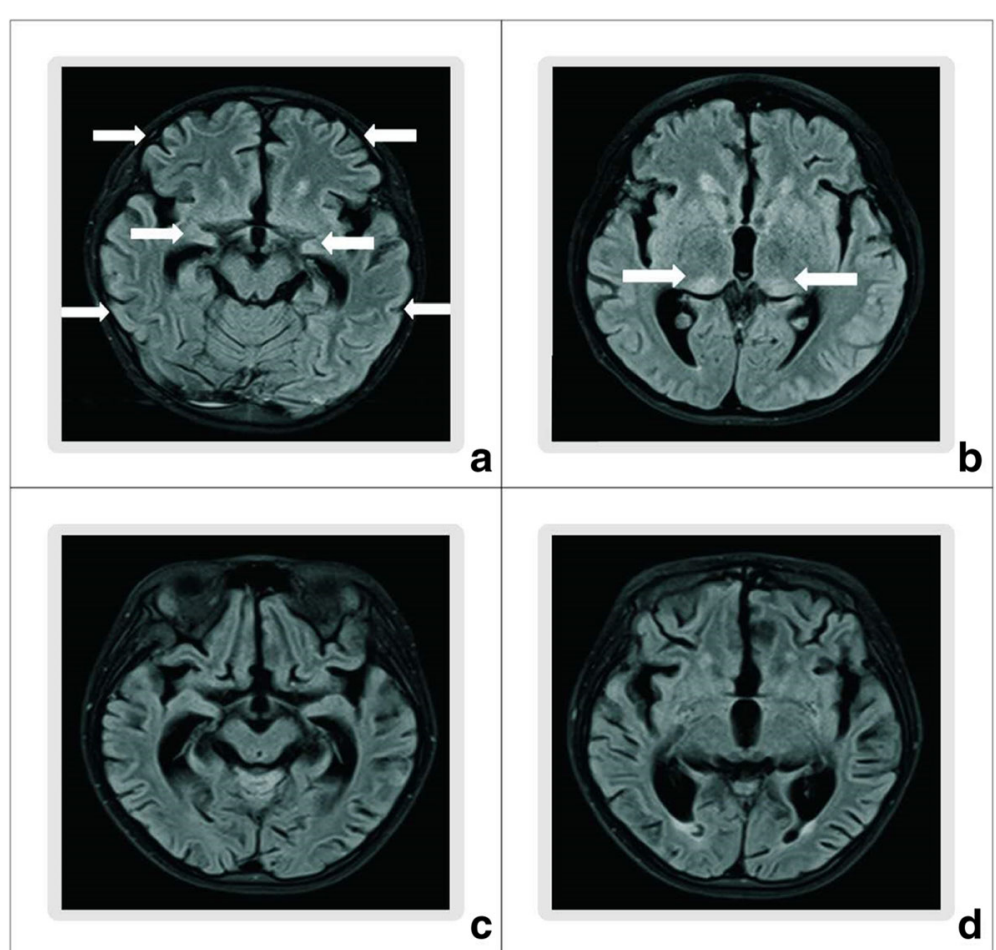

Fig. 2 MRI findings of the brains performed at acute $(\mathbf{a}, \mathbf{b})$ and chronic (c, d) stages upon seizure onset. $\mathbf{a}, \mathbf{b}$ Axial images showing abnormal T2 flair signal involving the frontal and temporal lobes and bilateral hippocampus and thalami (arrows). c, d Axial T2 flair images showing global cerebral atrophy

mild and could be relieved by symptomatic treatment which didn't lead to the discontinuation of KD. Another patient got arrhythmia at 27 days of use of KD, which was discontinued for patient safety reason (Table 2).

\section{Discussion}

SRSE may present with contradictory clinical manifestations and EEG findings, especially in children. Different SE types and various EEG patterns may co-exist in one patient. SE may occur at any stage of the disease process during or after the withdrawal of anaesthetic therapy. All patients in this study were failed to be controlled by the AEDs and anaesthetic therapy. Therefore, it is necessary to monitor seizures by using CEEG especially for NCSE.

FIRES is a life-threatening medical emergency that affects normal children after a febrile illness, and it is a major cause of SRES in paediatric intensive care units (PICUs). SE in FIRES regularly lasts for a few weeks and may cause death or progress to drug-resistant epilepsy associated with severe intellectual disabilities. The aetiologies and pathogenetic mechanisms of FIRES remain largely unknown. CSF tests showed no signs of viral infection or autoimmunity in most cases. Recent studies showed that some gene mutations in febrile epilepsy syndrome, such as mutations of PCDH19, SCN1A and $P O L G$, are not responsible for FIRES [8]. Genetic testing result of six patients through gene-targeted sequencing was also negative in the present study.

MRI findings in the acute stage may be normal. Some patients may show abnormal signal in bilateral hippocampal regions, in brain gray matter and deep grey matter nuclei. A previous study also reported MRI findings of bilateral mesial temporal or general atrophy in chronic stage, and T2 hyperintensities were seen in almost half of the cases [9]. The 10 patients with SRSE were consistent with the clinical features of FIRES. Among them, nine cases ultimately evolved into refractory epilepsy and presented with mild to severe intellectual disabilities with obvious MRI changes in the early and late stages, only one patient received favorable outcome with normal MRI. Therefore, MRI change could be used as a prognostic indicator for FIRES.

The first administration of KD may be traced back to the 1920s and was used as a treatment option for various types of refractory epilepsies in children and young adults [10-13]. Previous studies reported the successful application of KD to treat SRSE caused by FIRES or different encephalopathies, and significant clinical responses occurred within 1-19 days following the onset of KD in paediatric cases $[9,11,14]$. Five of the 10 patients in the present study recovered from SE within 6-15 days of the initiation of $\mathrm{KD}$, which was consistent with previous 


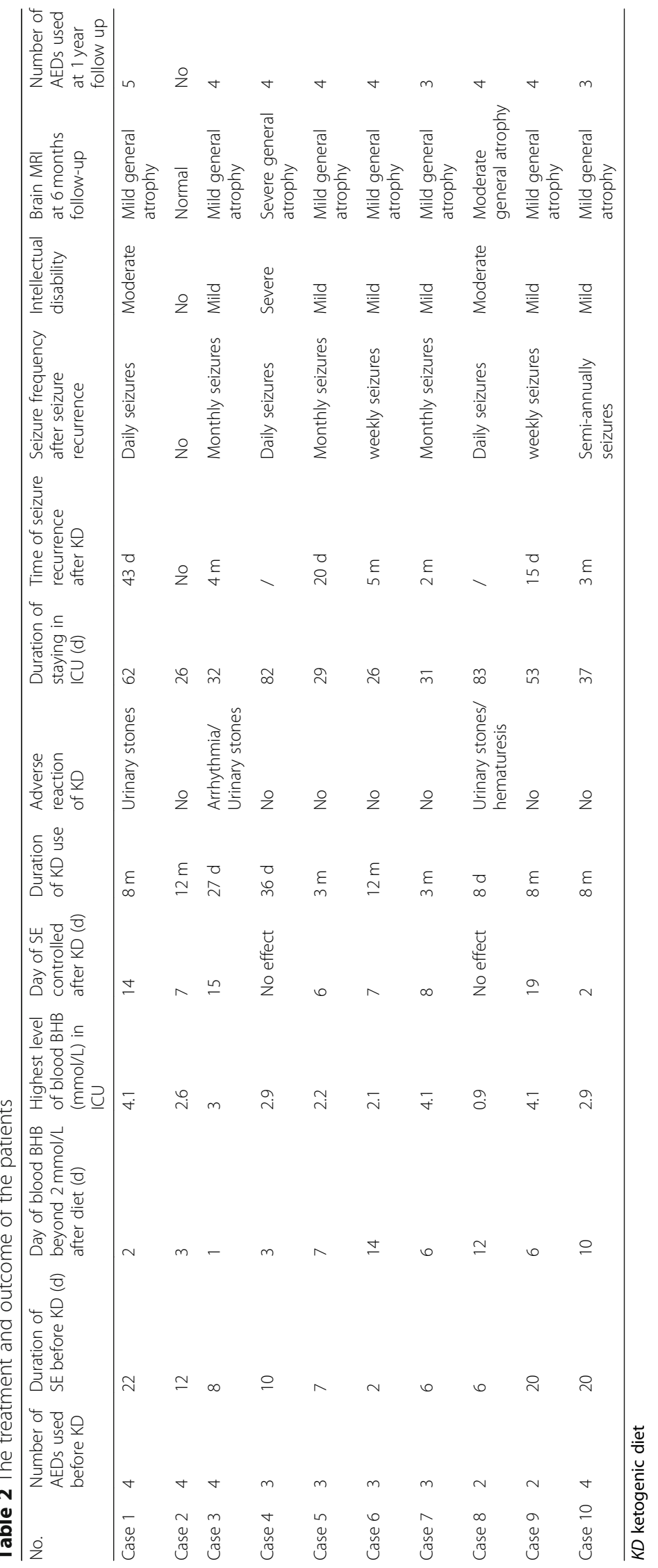


studies. To our knowledge, standardized evaluation criteria for the efficacy of KD on SE are not established according to previous studies [11,12,15-18]. The results of the present study are helpful to precisely evaluate the efficacy of KD through CEEG and aEEG monitoring rather than clinical manifestations, which are difficult to differentiate and standardize.

Previous studies have reported the adverse effects of $\mathrm{KD}$, including ketoacidosis, hypophosphatemia, hypokalaemia, high levels of triglycerides and pancreatitis [12-16]. In the present study, one subject (case 3) experienced an obvious reduction in seizure activities. KD discontinued because of ventricular fibrillation on the 27th day. He had no history of heart disease before KD treatment, and his family history was negative. Therefore, arrhythmia cannot be excluded as a side effect of KD. KD administration was significantly correlated with prolonged QT in a previous study [19]. Therefore, continuous electrocardiogram monitoring and routine surveillance of clinical and biochemical testing are necessary during KD therapy for these patients. Renal calculi occurs in $3-7 \%$ of refractory epileptic children following KD treatment [20], but the incidence of urinary calculi was approximately $30 \%$ (3 out of 10 patients) in our study, which is significantly higher than that in the previous literature. The prolonged bed rest may contribute to the formation of urinary calculi in these patients.

Although KD can control SE effectively, the occurrence of chronic epilepsy cannot be prevented. KD effectively controlled SE in eight cases in the present study. However, seizures recurred 15 days to 5 months after KD treatment and inevitably progressed to refractory epilepsy in seven patients after the use of KD for 3-12 months. These results indicated that there were unknown additional mechanisms involved in epileptogenesis after FIRES, and further studies are necessary.

Notably, several previous studies denied the role of urinary ketones in evaluations of the efficacy of $\mathrm{KD}$, but they affirmed the compliance of urinary ketones [21]. In contrast to previous studies that used urinary ketone values to monitor the efficacy and adverse effects of $\mathrm{KD}$, we introduced blood BHB instead of urinary ketone, as ketosis found in the urine might not be a better indicator of efficacy and fewer measurements of BHB was required to assess the extent of ketosis. Goal blood BHB levels were rapidly obtained within 1-3 days following KD onset after fasting for 3 days, and hypoglycaemia did not occur in our series. The limitations of this study can be summed up in two points: Firstly, the sample size was small. Secondly, it is lack of urinary ketone results to evaluate the relationship between blood BHB and seizure treatment outcome. Further studies should enrol more patients and measure the urine ketone results to confirm the relationship between them.

\section{Conclusion}

Our study is helpful to evaluate $\mathrm{KD}$ as a safe, effective treatment. KD treatment in paediatric cases with SRSE in FIRES should be considered early.

\section{Abbreviations \\ KD: Ketogenic diet; SRSE: Super-refractory status epilepticus; FIRES: Febrile infection-related epilepsy syndrome; CEEG: Continuous encephalography; aEEG: amplitude-integrated electro-encephalography; NORSE: New-onset refractory status epilepticus; MRI: Magnetic resonance imaging; CSE: Convulsive status epilepticus; NCSE: Nonconvulsive status epilepticus; CSF: Cerebrospinal fluid; AEDs: Anti-epileptic drugs; FS: Febrile seizure; GCS: Glasgow Coma Scale; ICU: Intensive care unit}

\section{Acknowledgements}

We sincerely thank the patients and their families for participating in the study. We would like to thank AJE (www.aje.com) for English language editing.

\section{Authors' contributions \\ $\mathrm{XHW}$ is the first author and performed data analysis and interpretation, and drafted the manuscript. XG and GPL performed the analysis and interpretation of clinical data. ZYL performed diet therapy and data analysis. SZZ and YW conceived the idea for the study and supervised the study. YFZ is the corresponding author and conceived the idea for the study, designed the study, supervised the study, interpreted the data, and drafted and revised the manuscript content. All authors read and approved the final manuscript.}

\section{Authors' information}

Not applicable.

\section{Funding}

"Omics-based precision medicine of epilepsy" entrusted by Key Research Project of the Ministry of Science and Technology of China (Grant No.2016YF(0904400).

\section{Availability of data and materials}

The datasets analysed during the current study are available from the corresponding author on reasonable request.

\section{Ethics approval and consent to participate}

The local ethics committee of Children's Hospital of Fudan University approved the study protocol, and parents gave written informed consent for study participation.

\section{Consent for publication}

All authors agree to publish the study.

\section{Competing interests}

The authors declare that they have no competing interests.

\section{Author details}

${ }^{1}$ Department of Neurology, Children's Hospital of Fudan University, Shanghai 201102, China. 'Department of Intensive Care Unit, Children's Hospital of Fudan University, Shanghai 201102, China. ${ }^{3}$ Department of Clinical Nutrition, Children's Hospital of Fudan University, Shanghai 201102, China.

Received: 18 June 2019 Accepted: 6 March 2020

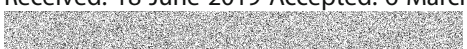

References

1. Ferlisi M, Shorvon S. The outcome of therapies in refractory and superrefractory convulsive status epilepticus and recommendations for therapy. Brain. 2012;135:2314-28. 
2. Rossetti AO, Reichhart MD, Schaller MD, Despland PA, Bogousslavsky J. Propofol treatment of refractory status epilepticus: a study of 31 episodes. Epilepsia. 2004;45:757-63.

3. Claassen J, Hirsch LJ, Emerson RG, Bates JE, Thompson TB, Mayer SA. Continuous EEG monitoring and midazolam infusion for refractory nonconvulsive status epilepticus. Neurology. 2001;57:1036-42.

4. Young GB, Jordan KG, Doig GS. An assessment of nonconvulsive seizures in the intensive care unit using continuous EEG monitoring: an investigation of variables associated with mortality. Neurology. 1996;47:83-9.

5. Gaspard N, Hirsch L, Sculier C, Loddenkemper T, van Baalen A, Lancrenon J, et al. New-onset refractory status epilepticus (NORSE) and febrile infectionrelated epilepsy syndrome (FIRES): state of the art and perspectives. Epilepsia. 2018;59(4):745-52.

6. Hellstrom-Westas $L$, Rosen I. Continuous brain-function monitoring:state of the art in clinical practice. Semin Fetal Neonatal Med. 2006:11:503-11.

7. Leitinger M, Beniczky S, Rohracher A, Gardella E, Kalss G, Qerama E, et al. Salzburg consensus criteria for non-convulsive status epilepticus-approach to clinical application. Epilepsy Behav. 2015;49:158-63.

8. Appenzeller S, Helbig I, Stephani U, Häusler M, Kluger G, Bungeroth M, et al. Febrile infection-related epilepsy syndrome (FIRES) is not caused by SCN1A, POLG, PCDH19 mutations or rare copy number variations. Dev Med Child Neurol. 2012:54:1144-8.

9. Howell KB, Katanyuwong K, Mackay MT, Bailey CA, Scheffer IE, Freeman JL, et al. Long-term follow-up of febrile infection-related epilepsy syndrome. Epilepsia. 2012;53:101-10.

10. Cobo NH, Sankar R, Murata KK, Sewak SL, Kezele MA, Matsumoto JH. The ketogenic diet as broad-spectrum treatment for super-refractory pediatric status epilepticus: challenges in implementation in the pediatric and neonatal intensive care units. J Child Neurol. 2015;30:259-66.

11. Bayrlee A, Ganeshalingam N, Kurczewski L, Brophy GM. Treatment of superrefractory status epilepticus. Curr Neurol Neurosci Rep. 2015;15:66.

12. Wilmshurst JM, Gaillard WD, Vinayan KP, Tsuchida TN, Plouin P, Van Bogaert $P$, et al. Summary of recommendations for the management of infantile seizures: task force report for the ILAE Commission of Pediatric. Epilepsia. 2015:56:1185-7.

13. Nabbout R, Mazzuca M, Hubert P, Peudennier S, Allaire C, Flurin V, et al. Efficacy of ketogenic diet in severe refractory status epilepticus initiating fever induced freactory epileptic encephalopathy in school age children (FIRES). Epilepsia. 2010;51:2033-7.

14. Sort R, Born AP, Pedersen KN, Fonsmark L, Uldall P. Ketogenic diet in 3 cases of childhood refractory status epilepticus. Eur J Paediatr Neurol. 2013;17:531-6.

15. Fung EL, Chang SK, Yam KK, Yau PY. Ketogenic diet as a therapeutic option in super-refractory status epilepticus. Pediatr Neonatol. 2015;56:429-31.

16. Caraballo RH, Flesler S, Armeno M, Fortini S, Agustinho A, Mestre G, et al. Ketogenic diet in pediatric patients with refractory focal status epilepticus. Epilepsy Res. 2014;108:1912-6.

17. Appavu B, Vanatta L, Condie J, Kerrigan JF, Jarrar R. Ketogenic diet treatment for pediatric super-refractory status epilepticus. Seizure. 2016:41:62-5.

18. O'Connor SE, Ream MA, Richardson C, Mikati MA, Trescher WH, Byler DL, et al. The ketogenic diet for the treatment of pediatric status epilepticus. Pediatr Neurol. 2014;50:101-3.

19. Best TH, Franz DN, Gilbert DL, Nelson DP, Epstein MR. Cardiac complications in pediatric patients on the ketogenic diet. Neurology. 2000;54(12):2328-30.

20. Kossoff EH, Zupec-Kania BA, Amark PE, Ballaban-Gil KR, Christina Bergqvist AG, Blackford R, et al. Optimal clinical management of children receiving the ketogenic diet: recommendations of the international Ketogenic diet study group. Epilepsia. 2009;50:304-17.

21. Rv D, Lambrechts D, Verschuure P, Hulsman J, Majoie M. Blood betahydroxybutyrate correlates better with seizure reduction due to ketogenic diet than do ketones in the urine. Seizure. 2010;19:36-9.

\section{Ready to submit your research? Choose BMC and benefit from:}

- fast, convenient online submission

- thorough peer review by experienced researchers in your field

- rapid publication on acceptance

- support for research data, including large and complex data types

- gold Open Access which fosters wider collaboration and increased citations

- maximum visibility for your research: over $100 \mathrm{M}$ website views per year

At BMC, research is always in progress.

Learn more biomedcentral.com/submissions 